\title{
Influence of flavonoids' lipophilicity on platelet aggregation
}

\author{
IVANA BABIĆ ${ }^{1}$ \\ MIRZA BOJIĆ2,* \\ ŽELJAN MALES̆ ${ }^{3}$ \\ RENATA ZADRO 4,5 \\ KORALJKA GOJČETA ${ }^{1}$ \\ IVAN DUKA ${ }^{3}$ \\ HRVOJE RIMAC ${ }^{2}$ \\ IRENA JUKIĆ \\ ${ }^{1}$ Croatian Institute of Transfusion \\ Medicine, HR-10000 Zagreb, Croatia \\ 2 University of Zagreb Faculty of \\ Pharmacy and Biochemistry \\ Department of Medicinal Chemistry \\ HR-10000 Zagreb, Croatia \\ ${ }^{3}$ University of Zagreb Faculty of \\ Pharmacy and Biochemistry \\ Department of Pharmaceutical Botany \\ HR-10000 Zagreb, Croatia \\ ${ }^{4}$ University of Zagreb Faculty of \\ Pharmacy and Biochemistry \\ Department of Clinical Chemistry \\ and Haematology, HR-10000 Zagreb \\ Croatia \\ ${ }^{5}$ University Hospital Centre Zagreb \\ Department of Laboratory Diagnostics \\ HR-10000 Zagreb, Croatia
}

Accepted July 23, 2019

Published online September 5, 2019

\begin{abstract}
Flavonoids are natural polyphenolic compounds present in a wide spectrum of plants that have a beneficial effect on human health. In the context of cardiovascular diseases related to plaque and thrombus formation, flavonoids exhibit an antiaggregatory effect. Previously, it has been reported that all tested flavonoids exhibit an antiaggregatory effect on platelet aggregation when measured by impedance aggregometry on whole blood, in the test of aggregation induced by adenosine diphosphate (ADP). As not all flavonoids have the same targets within signaling pathways, an assumption of a common non-specific mechanism related to lipophilicity is to be considered. To test this hypothesis, reverse-phase thin layer chromatography was used to assess the lipophilicity of flavonoids; impedance aggregometry was used for testing of platelet aggregation and flow cytometry to monitor the influence of flavonoids on platelet activation. Lipophilicity analysis showed a highly negative correlation of $\log P$ and $M I N a A C$ for groups of flavones and flavanones. As determined by flow cytometry, the exposition of receptors necessary for the promotion of platelet activation and primary clot formation was diminished, i.e., lowered expression of the activated form of integrin $\alpha \operatorname{IIb} \beta 3$ was observed in the presence of flavanone. Platelet membrane stabilization by flavonoids as a mechanism of antiaggregatory effect has been supported by impedance aggregometry experiments when specific inhibitors of platelet aggregation signaling pathways (U73122, indomethacin, verapamil) were used in the presence of a weak (ADP) and a strong (TRAP-6) agonist of aggregation. While individual flavonoids can have specific targets within aggregation signaling pathways, all flavonoids share a common non-specific mechanism of platelet aggregation inhibition related to their lipophilicity and membrane stabilization that, to some extent, contributes to their antiaggregatory effect.
\end{abstract}

Keywords: flavonoids, platelets, lipophilicity, flow cytometry, antiaggregatory activity

Flavonoids are natural polyphenolic compounds present in a wide spectrum of plants. One of the prominent and well documented beneficial effects on human health that has been confirmed in epidemiological studies is known as French paradox, i.e., although

\footnotetext{
*Correspondence; e-mail: mbojic@pharma.hr
} 
French population consumes fat-rich diet, incidence of cardiovascular diseases is lower than expected as their diet is also rich in polyphenols $(1,2)$. Based on their accessibility to humans through the dietary intake of fruits, vegetables and some beverages, flavonoids have been a topic of research for many years including their possible use as lead compounds for novel pharmaceutical agents. Many in vitro studies on plant and fruit extracts or on pure flavonoid compounds confirm antithrombotic, especially antiplatelet, effect in dose depending manner although without revealing the mechanism of action (3-5). In regard to cardiovascular diseases related to plaque and thrombus formation, flavonoids exhibit the antiaggregatory effect. For some flavonoids, this is attributed to the inhibition of cyclooxygenase, lipoxygenase, tyrosine-kinase, phosphodiesterase or phospholipase which participate in platelet signaling pathway (6-8).

Lipophilicity of flavonoids has been a subject of studies exploring flavonoid-membrane interactions. According to the results presented in previous studies, flavonoids are positioned within the membrane depending on their polarity, with more hydrophobic ones which penetrate deeper in the core of membrane bilayer, interact with membrane transporter proteins, and decrease membrane fluidity $(9,10)$. Platelet membrane is the best example of membrane fluidity; it functions as a dynamic structure involved in processes of activation and aggregation. These processes depend strongly on phospholipid transverse distribution in resting and activated state, and processes in the inner leaflet of platelet membrane (11).

Work of Lipinski et al. (12) represents the milestone for determination of the relationship between pharmacokinetic and physicochemical parameters by establishing "rule-offive" and uses $\log P$ as a measure of the compound's lipophilicity. Partition coefficient $(P)$ represents a ratio between the concentration of the non-ionized form of the compound in the $n$-octanol-water system expressed in $\log$ arithmic scale $(\log P)$. While the shake-flask method is the gold standard for $\log P$ determination, lipophilicity is nowadays more often determined indirectly by liquid chromatography (13). The simple approach is the determination by planar chromatography that uses chromatographic parameter $R_{\mathrm{M}}$ (parameter calculated based on the retention factor of substance) as a surrogate measurement of lipophilicity. To bypass laborious and time-consuming experimental methods, several computational approaches for the prediction of lipophilicity $(\log P)$ have been developed based on pool of predefined atoms, fragments and their contribution factors (14-16).

In the previous work by Bojić et al. (17) it has been shown that all tested flavonoids, without exception, exhibit the antiaggregatory effect on platelet aggregation induced by adenosine diphosphate (ADP). As not all flavonoids have the same targets within signaling pathways, an assumption of a common non-specific mechanism related to lipophilicity is to be considered. Thus, the aim of this study was to examine the flavonoids antiaggregatory mechanism based on lipophilicity and influence of flavonoids on platelet membrane rigidity/ fluidity.

\section{EXPERIMENTAL}

\section{Subjects}

Blood samples were collected at Croatian Institute of Transfusion Medicine (Zagreb, Croatia) in $4.5-\mathrm{mL}$ vacutainer tubes with tri-sodium citrate (3.8\%) as an anticoagulant. Fifty blood donors who were not on any antiaggregatory therapy gave written, informed consents before participating in research. The study was conducted according to Helsinki declaration and with approval of Ethics Committee of the Croatian Institute of Transfusion 
Medicine and the University of Zagreb Faculty of Pharmacy and Biochemistry. All measurements were conducted in two hours after the blood sample collection.

\section{Reagents}

Flavonoid aglycons used in this work are listed in Table I. Flavonoid solutions were prepared by dissolving standards in methanol (Kemig, Croatia) for the purpose of thinlayer chromatography analysis, while serial of doubling dilutions $\left(1 / 2^{\mathrm{n}}\right)$ in dimethyl sulfoxide (DMSO, Merck KgaA, Germany) in the concentration range of $500 \mathrm{mmol} \mathrm{L}^{-1}$ to 62 nmol L-1 was used for platelet aggregation analysis. ADP, collagen, TRAP-6, arachidonic acid (AA) and ristocetin were obtained from Roche Diagnostics (Germany), and saline (0.9 $\% \mathrm{NaCl}$ ) with and without calcium chloride from the Croatian Institute of Transfusion Medicine. Inhibitor of cyclooxygenase, indomethacin, and calcium channels blocker, verapamil hydrochloride, were purchased from Acros Organics (Thermo Fisher Scientific, Belgium) and inhibitor of phospholipase C (PLC), U73122 from Merck KgaA. Anti-integrin $\alpha \operatorname{IIb} \beta 3$ antibody (PAC-1) labelled with fluorescein isothiocyanate (FITC), anti-CD61 labelled with phycoerythrin (PE), FITC mouse IgM, $\kappa$ isotype control and PE mouse IgG1, $\kappa$ isotype control for flow cytometry experiments were purchased from Becton Dickinson (USA). Reverse-phase thin-layer chromatography was performed on Merck RP-18 F254S plates with a mobile phase consisting of water, acetonitrile and glacial acetic acid (last two solvents were obtained from Kemig, Croatia, while distilled water was prepared in house).

\section{Reverse-phase thin-layer chromatography and computational determination of lipophilicity}

Twenty flavonoid compounds were analyzed by reverse-phase thin-layer chromatography (RP-TLC) using acetonitrile/water/glacial acetic acid in a ratio 66:33:1 (V/V/V) as a mobile phase. After development, flavonoids were detected as dark spots on a chromatographic plate with the fluorescent indicator (wavelength $254 \mathrm{~nm}$ ). Based on retention factor, $R_{\mathrm{F}}$, chromatographic parameter $R_{\mathrm{M}}$ was calculated as follows $R_{\mathrm{M}}=\log \left[\left(1 / R_{\mathrm{F}}\right)-1\right]$.

Calculation of theoretical $\log P$ was performed using online applications Chemicalize (https://chemicalize.com), ChemSketch (http://www.chemspider.com), Molinspiration (https://www.molinspiration.com) and SwissADME (http://www.swissadme.ch). SwissADME is calculating $\log P$ in five different ways and expresses their consensus $\log P$ value. Values of experimentally determined chromatographic parameter $R_{\mathrm{M}}$ were correlated with calculated $\log P$ values using MS Office Excel (Microsoft, USA) and reported as correlation coefficient and adjusted squared correlation coefficient at a significance level of $p<0.05$.

\section{Impedance aggregometry}

Impedance aggregometry on whole blood was monitored on Multiplate ${ }^{\circledR}$ instrument (Roche Diagnostics, Germany) and was used for determination of minimum antiaggregatory concentration (MINaAC) of flavanone in the presence of a set of agonists (TRAP-6, ADP, collagen, AA, ristocetin) by previously described method (17). Briefly, the influence of serial doubling concentrations of flavanone on platelet aggregation was measured by using original Multiplate procedure as follows: $300 \mu \mathrm{L}$ of blood was incubated for 3 minutes at $37^{\circ} \mathrm{C}$ with 20 $\mu \mathrm{L}$ flavonoid and $300 \mu \mathrm{L}$ of saline-CaCl${ }_{2}$ preheated at $37^{\circ} \mathrm{C}$. Vehicle-DMSO (final concentration $3 \%$ ) was used as a negative control (untreated sample). Aggregation was induced by addition of $20 \mu \mathrm{L}$ of the agonist with final concentrations: $6.3 \mu \mathrm{mol} \mathrm{L}-1$ ADP, $3.1 \mu \mathrm{g} \mathrm{mL}-1$ collagen, $31 \mu \mathrm{mol} \mathrm{L}-1$ TRAP-6, $0.48 \mu \mathrm{mol} \mathrm{L}^{-1} \mathrm{AA}$, and $0.3 \mathrm{mg} \mathrm{mL}^{-1}$ ristocetin. MINaAC was 
determined in three independent measurements for treated and untreated sample using the Student's $t$-test. The same pre-defined Multiplate protocol was followed for the assessment of the combined effect of inhibitory drugs (U73122 hydrate, verapamil hydrochloride, indomethacin) and flavanone. Measurements were conducted with TRAP-6 as a strong and ADP as a weak agonist using 15 and $60 \times M I N a A C$ of flavanone to ensure repeatable inhibition effect on platelet aggregation. U73122 hydrate, verapamil hydrochloride, and indomethacin were

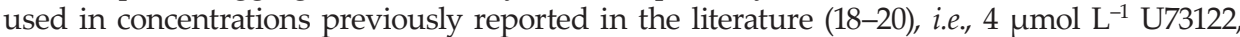
$82.5 \mu \mathrm{mol} \mathrm{L}-1$ verapamil and $0.21 \mu \mathrm{mol} \mathrm{L}-1$ indomethacin in platelet aggregation test induced with $30 \mu \mathrm{mol} \mathrm{L}{ }^{-1}$ TRAP-6, while in platelet aggregation test induced with $6.3 \mu \mathrm{mol} \mathrm{L}{ }^{-1}$ ADP concentrations were for U73122, verapamil and indomethacin $3.2 \mu \mathrm{mol} \mathrm{L}-1,52 \mu \mathrm{mol} \mathrm{L}^{-1}$ and $0.21 \mu \mathrm{mol} \mathrm{L}^{-1}$, resp. Results of aggregation for untreated sample (DMSO, $0.8 \%$ ), flavanone treated, inhibitor drug-treated, and for the sample treated with the combination of flavanone and inhibitor drug were statistically assessed using ANOVA.

\section{Flow cytometry}

Platelet-rich plasma (PRP) was obtained by centrifugation of freshly drawn citrate blood samples at $150 \mathrm{~g}$ for $10 \mathrm{~min}$. Platelet concentration was adjusted to 20.000 per $\mu \mathrm{L}$ with phosphate-buffered saline ( $\mathrm{pH}$ 7.4). One $\mathrm{mL}$ of PRP was pre-incubated with three dilutions of flavanone $\left(30,244\right.$ and $\left.488 \mu \mathrm{mol} \mathrm{L}^{-1}\right)$ or with the vehicle as a control (DMSO, $0.8 \%$ ) for 15 minutes at $37^{\circ} \mathrm{C}$. An aliquot of $72 \mu \mathrm{L}$ treated sample (flavanone, DMSO) was mixed with agonist TRAP-6 (final concentration $21 \mu \mathrm{mol} \mathrm{L}^{-1}$ ) and the excess amount of anti-CD61 (PE) to mark platelet population and PAC-1 FITC to monitor the activated form of integrin $\alpha \operatorname{IIb} \beta 3$. The sample was incubated in the dark at $37^{\circ} \mathrm{C}$, for $30 \mathrm{~min}$. Flow cytometry runs were performed on FC500, Beckman Coulter (France). To distinguish platelet cell population based on cell size and granularity, forward vs. side scatter was used collecting 10.000 CD61 positive events. Isotype controls FITC labelled mouse IgM $\kappa$, and IgG1 PE were used to monitor the level of non-specific background signal. Activated platelets were assessed as the percentage of platelets with positive activation-dependent platelet surface marker PAC-1 FITC in CD61 positive cells gate. Results were obtained as a measurement in triplicate and tested with paired Student's $t$-test for significance. To confirm the concentration level of flavanone which caused statistically significant inhibition of platelet activation, measurements were repeated on five independent samples in triplicate.

\section{Statistical analysis}

The antiaggregatory effect of flavanone and inhibitor drugs were assessed with oneway ANOVA test with post hoc Tukey analysis for multiple comparison. For comparison of $R_{\mathrm{M}}$ and $\log P$ linear regression analysis was performed. Paired Student's $t$-test was used for the estimation of $\mathrm{MINaAC}$ and platelet activation inhibition measured by flow cytometry. If not otherwise stated, statistical analysis was performed within $\mathrm{R}$ v2.8.1 environment (R-project, Austria).

\section{RESULTS AND DISCUSSION}

\section{Lipophilicity of flavonoids}

To assess the lipophilicity of flavonoids reverse-phase thin-layer chromatography was conducted on a series of 20 compounds. Chromatographic parameter, $R_{\mathrm{M}}$, the surrogate of 
Table I. Experimental (chromatographic parameter $\mathrm{R}_{M}$ ), calculated parameters $(\log \mathrm{P})$ of flavonoid lipophilicity and $\mathrm{MINaC}$

\begin{tabular}{|c|c|c|c|c|c|c|c|c|c|c|c|}
\hline Flavonoid & $R_{\mathrm{M}}$ & 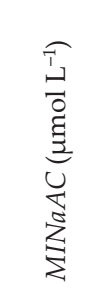 & 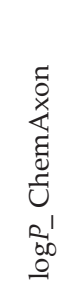 & 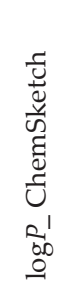 & 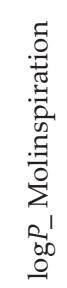 & 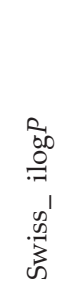 & $\begin{array}{l}\sum_{00}^{\infty} \\
0 \\
\frac{0}{x} \\
\infty^{\prime} \\
\infty_{3}^{\infty} \\
\text { के }\end{array}$ & 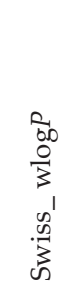 & 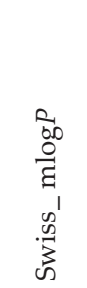 & 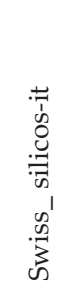 & 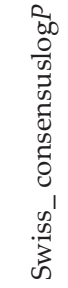 \\
\hline \multicolumn{12}{|l|}{ Flavones } \\
\hline Flavone $^{b}$ & 0.3892 & 3.815 & 2.97 & 3.56 & 3.74 & 2.55 & 3.56 & 3.46 & 2.27 & 4.04 & 3.18 \\
\hline 6-Hydroxyflavone ${ }^{c}$ & -0.0378 & 0.954 & 2.66 & 3.72 & 3.23 & 2.28 & 3.62 & 3.17 & 1.66 & 3.52 & 2.85 \\
\hline 7-Hydroxyflavone ${ }^{c}$ & -0.0884 & 15.259 & 2.66 & 3.32 & 3.23 & 2.22 & 3.62 & 3.17 & 1.66 & 3.52 & 2.84 \\
\hline Tangeretin $^{\mathrm{d}}$ & 0.4202 & 30.518 & 2.18 & 2.66 & 3.78 & 3.71 & 3.04 & 3.5 & 0.63 & 4.21 & 3.02 \\
\hline Tectchrysine $e^{\mathrm{e}}$ & 0.5202 & 0.954 & 3.16 & 3.13 & 3.48 & 2.88 & 3.85 & 3.17 & 1.33 & 3.52 & 2.95 \\
\hline Chrysine dimethyl ether ${ }^{\mathrm{e}}$ & 0.5202 & 1.907 & 3.01 & 3.27 & 3.75 & 2.95 & 3.62 & 3.48 & 1.57 & 4.04 & 3.13 \\
\hline Chrysine $^{b}$ & 0.0126 & 3.815 & 3.01 & 2.88 & 2.94 & 2.27 & 3.52 & 2.87 & 1.08 & 3.02 & 2.55 \\
\hline Acacetin $^{\mathrm{d}}$ & 0.0378 & 3.815 & 2.85 & 3.15 & 3.00 & 2.56 & 3.35 & 2.88 & 0.77 & 3.03 & 2.52 \\
\hline Diosmetin $^{\mathrm{e}}$ & -0.3590 & 7.629 & 2.55 & 3.10 & 2.28 & 2.47 & 3.1 & 2.59 & 0.22 & 2.55 & 2.19 \\
\hline Apigenin $^{\mathrm{b}}$ & -0.3892 & 3.815 & 2.71 & 2.10 & 2.46 & 1.89 & 3.02 & 2.58 & 0.52 & 2.52 & 2.11 \\
\hline Luteolin $^{\mathrm{e}}$ & -0.5942 & 7.629 & 2.40 & 2.40 & 1.97 & 1.86 & 2.53 & 2.28 & -0.03 & 2.03 & 1.73 \\
\hline \multicolumn{12}{|l|}{ Flavonols } \\
\hline 3,6-Dihydroxyflavone ${ }^{c}$ & -0.0378 & 0.119 & 2.42 & 3.64 & 2.94 & 1.97 & 3.05 & 2.87 & 1.08 & 3.02 & 2.4 \\
\hline 3,7-Dihydroxyflavone ${ }^{c}$ & -0.0631 & 1.907 & 2.42 & 3.27 & 2.94 & 2.01 & 3.05 & 2.87 & 1.08 & 3.02 & 2.4 \\
\hline Galangin ${ }^{\mathrm{b}}$ & 0.0000 & 122.070 & 2.76 & 2.83 & 2.65 & 2.08 & 2.25 & 2.58 & 0.52 & 2.52 & 1.99 \\
\hline Rhamnetin $^{\mathrm{d}}$ & -0.2083 & 0.954 & 2.30 & 2.58 & 2.22 & 2.23 & 1.87 & 2.29 & -0.31 & 2.06 & 1.63 \\
\hline Isorhamnetin $^{\mathrm{d}}$ & -0.3203 & 7.629 & 2.30 & 1.76 & 1.99 & 2.35 & 1.87 & 2.29 & -0.31 & 2.06 & 1.65 \\
\hline Quercetin ${ }^{b, f}$ & -0.5863 & 15.259 & 2.77 & 2.07 & 1.68 & 1.63 & 1.54 & 1.99 & -0.56 & 1.54 & 1.23 \\
\hline \multicolumn{12}{|l|}{ Flavanons } \\
\hline Pinocembrin-7-methylether ${ }^{\mathrm{e}}$ & 0.3100 & 0.954 & 3.28 & 4.11 & 3.13 & 2.75 & 3.20 & 2.78 & 1.52 & 3.06 & 2.66 \\
\hline Pinocembrin $^{e}$ & -0.1076 & 15.259 & 3.14 & 3.93 & 2.60 & 2.11 & 2.88 & 2.48 & 1.27 & 2.55 & 2.26 \\
\hline Hesperetin $^{\mathrm{b}}$ & -0.4536 & 1.907 & 2.68 & 2.90 & 1.94 & 2.24 & 2.60 & 2.19 & 0.41 & 2.08 & 1.91 \\
\hline Flavanone $^{\mathrm{b}}$ & 0.1811 & 0.062 & 3.10 & 3.62 & 3.18 & 2.41 & 3.14 & 3.07 & 2.47 & 3.57 & 2.93 \\
\hline
\end{tabular}

${ }^{a} \mathrm{MINaAC}$ represents minimal antiaggregatory concentration (taken from reference 17).

Flavonoids purchased from: ${ }^{\mathrm{b}}$ Merck KgaA, Germany, ${ }^{\mathrm{c}}$ ChromaDex, USA, ${ }^{\mathrm{d}}$ BioChemika, Switzerland, ${ }^{\mathrm{e}}$ Extrasynthese, France.

${ }^{\mathrm{f}}$ In the form of quercetin dihydrate.

$\log P$, was in the range from -0.59 to 0.52 . The highest $R_{\mathrm{M}}$ values were obtained for tectochrysin and chrysin dimethyl ether, and the lowest for luteolin (Table I). 
Table II. Results of regression analysis for chromatographic parameter $\mathrm{R}_{M}$ and logP for flavonoids from Table I

\begin{tabular}{lccc}
\hline Application & Adjusted $R^{2}$ & Multiple $R$ & $p$-value \\
\hline $\log P$ ChemAxon & 0.1556 & 0.4448 & 0.0434 \\
$\log P$ ChemSketch & 0.2605 & 0.5454 & 0.009 \\
$\log P$ _Molinspiration & 0.8545 & 0.9283 & $<0.001$ \\
Swiss_ilogP & 0.5649 & 0.7659 & $<0.001$ \\
Swiss_xlogP3 & 0.4011 & 0.6566 & 0.0012 \\
Swiss_wlogP & 0.7269 & 0.8605 & $<0.001$ \\
Swiss_mlogP & 0.4678 & 0.7032 & $<0.001$ \\
Swiss_silicos-it & 0.7530 & 0.8748 & $<0.001$ \\
Swiss_consensuslog $P$ & 0.7315 & 0.8631 & $<0.001$ \\
\hline
\end{tabular}

Computational values of $\log P$ determined in Chem Axon were in the range of 2.18 to 3.28 with the highest value for pinocembrin-7-methylether. The same compound had the highest value of ChemSketch calculated $\log P$ (4.11). In the case of Molinspiration calculated $\log P$, the most lipophilic flavonoids were flavone (3.74), tangeretin (3.78) and techtochrysin (3.48). SwissADME calculates $\log P$ according to five different algorithms and expresses their consensus $\log P$ value. These values were the highest for flavone (3.18), tangeretin (3.02) and chrysin dimethyl ether (3.13) which is in accordance with experimentally determined $R_{\mathrm{M}}$ values.

Results of linear regression (Table II) for multiple $\log P$ and $R_{\mathrm{M}}$ showed strong positive correlation $R=0.8631$ and $R=0.9283(p<0.001)$ for calculated SwissADME consensus $\log P$ and Molinspiration program, resp. ChemSketch and Chemicalize $\log P$ showed moderate positive correlation to chromatographic parameter $R_{\mathrm{M}} R=0.5454(p=0.009)$ and $R=0.4448$ $(p=0.0434)$.

Based on the strong positive correlation between $R_{\mathrm{M}}$ parameter and $\log P$ calculated by Molinspiration and SwissADME application and moderate positive correlation with ChemSketch and Chemicalize $\log P$, it can be concluded that chromatographic parameter $R_{\mathrm{M}}$ is a good surrogate of $\log P$ and a good predictor of flavonoid lipophilicity.

This approach was also previously used as a part of QSAR study of flavonoids as bacteriostatic agents against Escherichia coli, with the conclusion that high correlation between calculated $\log P(\mathrm{C} \log P)$ values and predicted $M I C_{50}$ implies that hydrophobicity plays a major role in the antibacterial activity of flavonoids by their interaction with membranes (21). When inhibition of Syk protein tyrosine kinase activity and human mast cells degranulation was studied on a set of flavonoids, a significant relationship was observed between compound $\log P$ and the observed inhibitory effect (22). $\log P$ was also included in global models for the evaluation of toxicity of flavonoid compounds in the metabolic environment of mammals (23). As a measure of lipophilicity, $\log P$ offers a very convenient way of predicting the possible extent of flavonoid action in vivo based on their interaction with biomembranes.

\section{Relationship between inhibition of platelet aggregation and lipophilicity of flavonoids}

For flavonoids listed in Table I previously tested in platelet aggregation assay induced by $\operatorname{ADP}(17), M I N a A C$ values were correlated with calculated $\log P$ (Table III). 
Table III. Correlations for $\log \mathrm{P}$ and minimal antiaggregatory concentration (MINaAC) of flavonoids

\begin{tabular}{lcccccc}
\hline Application & Flavone & Flavonols & Flavanons & $\begin{array}{c}\text { Flavons and } \\
\text { flavonols }\end{array}$ & $\begin{array}{c}\text { Flavons and } \\
\text { flavonons }\end{array}$ & $\begin{array}{c}\text { Flavonols and } \\
\text { flavonons }\end{array}$ \\
\hline $\log P$ ChemAxon & $-0.7576^{\mathrm{a}}$ & 0.6691 & 0.1599 & -0.0103 & $-0.5922^{\mathrm{a}}$ & 0.0724 \\
$\log P$ ChemSketch & -0.2667 & 0.0082 & 0.3013 & -0.1214 & -0.2005 & -0.1545 \\
$\log P$ _Molinspiration & 0.1908 & 0.1341 & -0.2218 & -0.0597 & 0.1498 & -0.0047 \\
Swiss_ilogP & 0.5384 & 0.0062 & -0.6678 & -0.0279 & 0.4078 & -0.1949 \\
Swiss_xlogP3 & -0.3550 & -0.1014 & -0.2666 & -0.3042 & -0.2297 & -0.2414 \\
Swiss_wlogP & 0.2406 & 0.0428 & -0.3616 & -0.1106 & 0.1785 & -0.0545 \\
Swiss_mlogP & -0.2522 & 0.0983 & -0.2195 & -0.1419 & -0.2668 & -0.1333 \\
Swiss_silicos-it & 0.2836 & 0.0303 & -0.3728 & -0.1070 & 0.1996 & -0.1066 \\
Swiss_consensuslog $P$ & 0.1157 & 0.0185 & -0.3645 & -0.1628 & 0.0542 & -0.1611 \\
\hline
\end{tabular}

a Statistically significant correlation $(p<0.05)$.

The relationship was tested on a whole set of flavonoids, as well as individual classes of flavonoids and their combinations (flavones, flavonols, flavanones, flavones and flavonols, flavones and flavanones, flavonols and flavanones). Statistically significant correlation was found between MINaAC and $\log P$ calculated by Chemicalize application: strong negative correlation $R=-0.7576(p=0.0069)$ for flavone group and moderate negative correlation $R=-0.5922$ ( $p=0.0200)$, for combined groups of flavone and flavanone. Van Dijk et al. (24) found that flavonols exhibited a substantially higher affinity for liposomes than flavanones attributing this result with a far more planar configuration of the flavonols in comparison with the tilted configuration of flavanones. Oteiza et al. (10) proposed lipophilicity of flavonoid molecule as a basis for flavonoid interactions with biomembranes as well as interactions of polyphenols at the surface of bilayers through hydrogen bonding that can act to reduce the access of deleterious molecules, thus protecting the structure and function of membranes. Results of the study by Arora et al. (25) on the modulation of membrane fluidity by flavonoids suggest that partition into the hydrophobic core causes a dramatic decrease in lipid fluidity of the membrane.

Our results are in concordance with the assumption that flavonoids have a tendency to localize in the membrane bilayer based on their lipophilicity. In that sense, flavonoids with higher lipophilicity have greater potency to interact with the membrane and consequently cause inhibition of platelet aggregation at a lower concentration, presumably by causing a decrease in lipid fluidity. Thus, the stabilized platelet membrane is less susceptible to the inducers of platelet aggregation. This is in accordance with high negative correlation observed between values of $\log P$ calculated by Chemicalize application with $M I N a A C$ values; flavonoids with higher values of $\log P$ have lower values of $M I N a A C$ and stronger antiplatelet effect.

When average values of $\log P$ (Chemaxon) for different classes of flavonoids were calculated (based on data in Table I), the following trend was observed: flavanones (2.50), flavonols (2.76), flavones (3.05). The same trend was observed when comparing antiaggregatory effect; the higher lipophilicity is, the more prone antiaggregatory effect is, i.e., MINaAC are lower: flavanons $\left(24.66 \mu \mathrm{mol} \mathrm{L}{ }^{-1}\right)$, flavonols $\left(7.75 \mu \mathrm{mol} \mathrm{L}{ }^{-1}\right)$, flavones $\left(4.55 \mu \mathrm{mol} \mathrm{L}^{-1}\right)$. 
Although this observation confirms objective of our work, it should be noted that not all classes of flavonoids were equally represented in our study (flavanones, $n=4$, flavonols, $n=6$, flavones, $n=11$ )

\section{Influence of flavonoids and antiplatelet drugs combinations on primary hemostasis}

When compared to our previous study, an antiaggregatory activity which was evaluated on a set of 30 flavonoids in the platelet-aggregation assay induced by ADP (17), flavanone showed much higher potency with MINaAC concentration in the nanomolar range $\left(63 \mathrm{nmol} \mathrm{L}^{-1}\right)$. As this was the lowest MINaAC that we have observed among all flavonoids present in our library of compounds, further studies were conducted using different commercially available inducers of platelet aggregation for impedance aggregometry, namely TRAP-6, collagen, ristocetin and arachidonic acid (Table IV).

Table IV. Effects of flavanone on platelet aggregation depending on the agonist used in impedance aggregation assay

\begin{tabular}{ccc}
\hline Agonist & $\begin{array}{c}M I N a A C \\
(\mu \mathrm{mol} \mathrm{L}-1)\end{array}$ & $p$-value \\
\hline ADP & 0.063 & 0.0366 \\
TRAP-6 & 2.000 & 0.0310 \\
Collagen & 0.500 & 0.0175 \\
Ristocetin & 0.500 & 0.0270 \\
AA & 0.500 & 0.0037 \\
\hline
\end{tabular}

AA - arachidonic acid, ADP - adenosine diphosphate, MINaAC minimal antiaggregatory concentration, TRAP-6 - thrombin receptor-activating peptide (hexapeptide)

In the herein presented study, MINaAC of $0.063 \mu \mathrm{mol} \mathrm{L}^{-1}$ was observed when a weak agonist ADP was used to induce aggregation. When TRAP-6, hexapeptide surrogate of thrombin, was used as an inducer of platelet aggregation MINaAC of $2 \mu \mathrm{mol} \mathrm{L}^{-1}$ was obtained. Similarly, stronger inducers of platelet aggregation required more flavanone to ob-

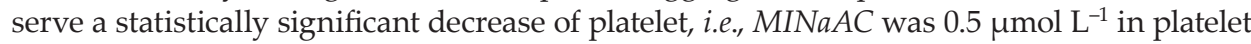
aggregation assays induced by collagen, ristocetin and AA.

Experiments on the combined inhibitory effect of flavanone and known inhibitors of platelet aggregation were designed on Multiplate ${ }^{\circledR}$ analyzer to assess their interactions and, to some extent, indicate a possible mechanism of flavanone action in the presence of strong or weak agonist. A PLC inhibitor U73122, calcium channels blocker verapamil and cyclooxygenase inhibitor indomethacin were used for this purpose. Each experiment included the determination of platelet aggregation of either flavanone or the inhibitor (known antiplatelet drug), or the combination of flavonoid and inhibitor. Platelet aggregation assays in this instance were induced by a strong (TRAP-6) or a weak (ADP) agonist of aggregation. To assess the statistical significance of different treatments one-way ANOVA with post hoc test was used for results assessment. The results of these analyses are shown in Fig. 1.

The statistically significant difference between treated samples was observed for the combination of U73122, an inhibitor of PLC, and flavanone, for both (TRAP-6, ADP) agonist applied (Fig. 1a). As expected, flavanone standard reduced platelet aggregation by $14 \%$, 
U73122 reduced platelet aggregation by $25 \%$ while the combination of the two reduced platelet aggregation by $35 \%$ in TRAP-6 induced platelet assay. However, the combined effect of flavanone and U73122 on platelet aggregation induced by ADP was a reduction of $50 \%$. Significant combined inhibitory effect of flavanone and U73122 on the first and most important enzyme in platelet activation cascade, even in the presence of a strong agonist, could be the consequence of specific inhibition of PLC-mediated signaling pathways upstream of PLC. As membrane fluidity is involved in the regulation of platelet activation, i.e., change of cell shape, pseudopodia formation and exposition of receptors that stimulate platelet aggregation (26, 27), our assumption is that flavonoids as lipophilic substances influence membrane fluidity by increasing membrane rigidity thus reducing the exposition of receptors at the membrane surface and subsequent activation of platelet aggregation signaling pathways. Experiments on $G$ protein-coupled receptor show that increased membrane fluidity stimulates an active form of the receptor (28). Flavanone can interact in a non-specific way with platelet membrane, and, thus, interfere in signal transduction of G-coupled proteins to PLC.

When combined with verapamil, flavanone showed a reduction of platelet aggregation induced by TRAP-6 (Fig. 1b). However, this effect was not observed when ADP was used as platelet aggregation inducer. As both pathways induced by ADP and TRAP-6 include calcium liberation from dense tubules it could be assumed that this is not a common target (29). Rather, upstream signaling pathways unique to TRAP-6 such as Rho/Rho-kinase could be possible targets of flavanone (29). The absence of combined inhibitory influence of flavanone and indomethacin regardless of the used agonist (Fig. 1c), indicates that the antiaggregatory effect of flavanone is not achieved downstream of cyclooxygenase 1.

a)

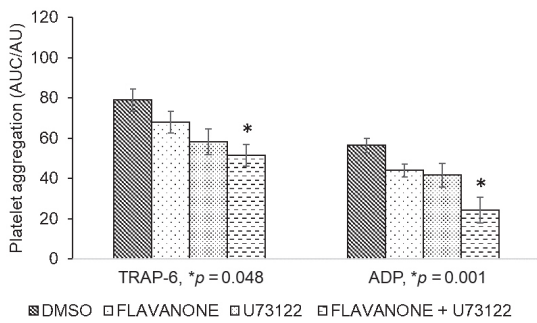

b)

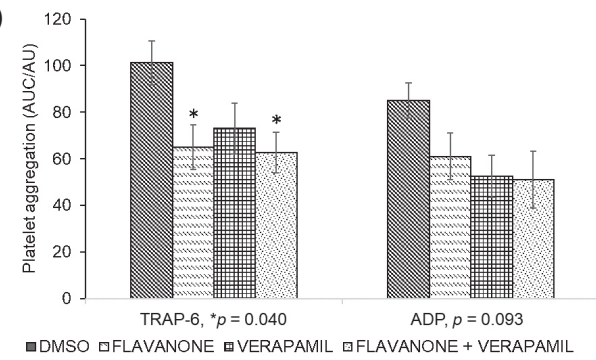

c)

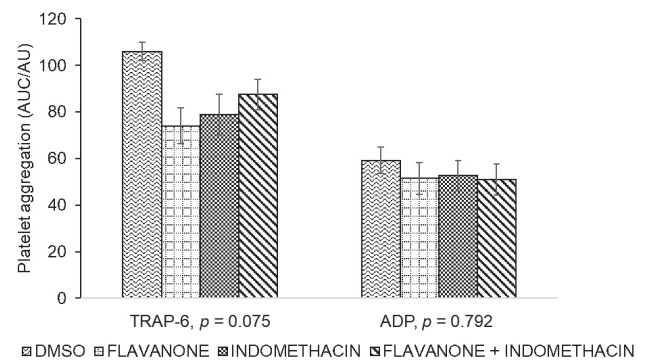

Fig. 1. Results of impedance aggregometry assays induced by a strong (TRAP-6) and a weak (ADP) agonist of aggregation for: a) flavanone and a phospholipase $\mathrm{C}$ inhibitor U73122, b) calcium channels blocker verapamil and c) cyclooxygenase inhibitor indomethacin, and flavanone and inhibitor drug combinations. Asterisks indicate statistical significance. 
Although the methodology used in these experiments lacks sensitivity to pinpoint precise target of flavanone, results obtained are in accordance with the presumption of the non-specific mechanism of platelet aggregation through the interaction with the membrane rigidity.

\section{Flow cytometry}

If flavanone influences membrane rigidity, expression of receptors on platelet surface will be reduced, e.g., by inhibiting transverse diffusion also known as flip-flop. To assess the expression of receptors in the presence and the absence of flavonoid, flow cytometry was used. Platelet activation trough modulation of inside-out signaling of integrin $\alpha \operatorname{IIb} \beta 3$ is significantly inhibited by flavanone (Figs. 2 and 3).

The observed effect is dose-dependent $(R=0.9897, p<0.05$, Fig. 2). An example of flow cytometry results is shown in Fig. 3: statistically significant reduction in the receptor exposition is achieved at higher concentrations of flavanone $\left(488 \mu \mathrm{mol} \mathrm{L}^{-1}\right)$ when compared to the untreated samples of the same participant $(p=0.0031)$. This result was confirmed subsequently by the analysis of five independent blood samples $(p=0.0018)$.

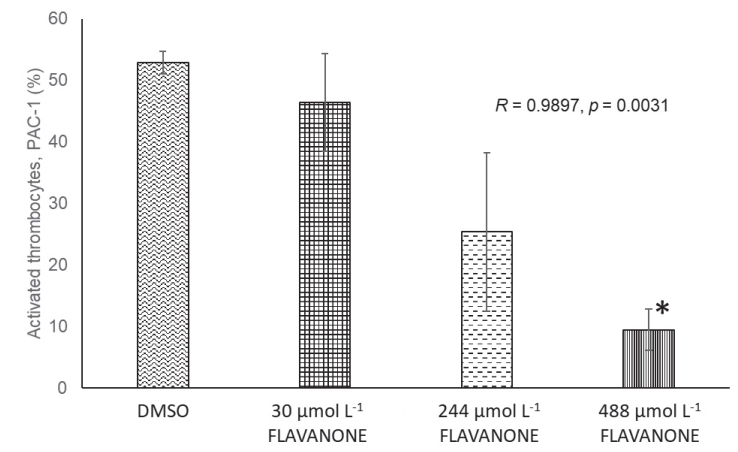

Fig. 2. Inhibitory effect of flavanone on platelet aggregation induced by TRAP-6 on platelet-rich plasma.
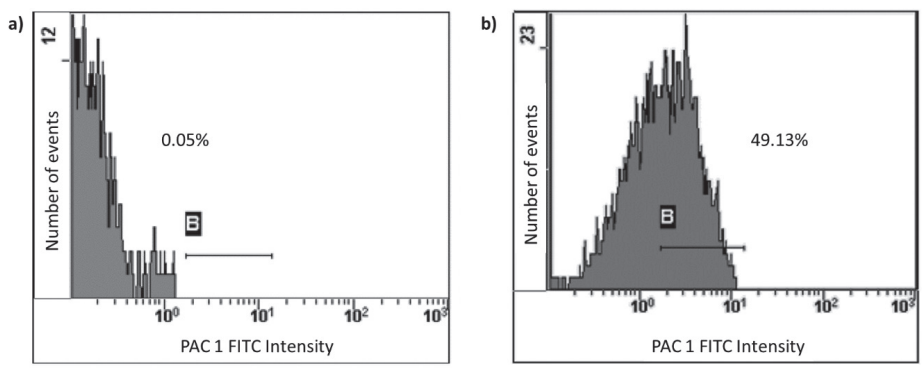

Fig. 3. Flow cytometry analysis of platelet activation induced by TRAP-6 in: a) absence and b) the presence of flavanone. Mean fluorescence intensity is measured using integrin $\alpha \operatorname{IIb} \beta 3$ antibody. 
Despite the noticeable effect, when compared with MINaAC observed in impedance aggregometry, only a rather high concentration of flavanone achieved significant inhibition of platelet activation in flow cytometry experiments. This could be due to the difference in the type of samples (impedance aggregometry uses whole blood while platelet-rich plasma is used for flow cytometry experiments), as well as experimental set up (concentration, sample manipulation, time, etc.). This is in accordance with the results of Ostertag et al. (30) and Vaiyapuri et al. (31) who also used higher concentrations of phenolic compounds to see measurable results in flow cytometry experiments. Although they present complementary methods, flow cytometry and impedance aggregometry comprise different aspects of platelet function and are therefore not interchangeable when agonist-induced platelet reactivity is assessed (32). As for methodology, impedance aggregometry, by performing in whole blood, enables other blood elements to influence platelet aggregation, therewith, functions under more physiological conditions (33).

\section{CONCLUSIONS}

In platelet aggregation assays induced by ADP, all flavonoids showed antiaggregatory activity (17), suggesting a non-specific mechanism of action related to their lipophilicity and stabilization of platelet membrane. Lipophilicity analysis showed a highly negative correlation between $\log P$ and $M I N a A C$ for groups of flavones and flavanones confirming the objective of our study. If so, the exposition of receptors necessary for the promotion of platelet activation and primary clot formation should be diminished. This was confirmed by flow cytometry in which lowered expression of the activated form of integrin $\alpha \operatorname{IIb} \beta 3$ was observed in the presence of flavanone. Platelet membrane stabilization by flavonoids as a mechanism of antiaggregatory effect has been supported by impedance aggregometry experiments when specific inhibitors of platelet aggregation signaling pathways were used in the presence of a weak and strong agonist of aggregation. While individual flavonoid can have specific targets within aggregation signaling pathways, flavonoids share a common non-specific mechanism of platelet aggregation inhibition related to their lipophilicity and membrane stabilization that, to some extent, contributes to their antiaggregatory effect.

Acknowledgements. - The authors would like to thank A. Jurak Begonja and J. Bingulac-Popović, for their helpful advices.

Acronyms, abbreviations, symbols. - AA - arachidonic acid, ADP - adenosine diphosphate, CD cluster of differentiation, $\operatorname{Clog} P$ - calculated $\log P$, FITC - fluorescein isothiocyanate, Ig - immunoglobulin, $\log P$ - logarithm of partition coefficient, MINaAC - minimal antiaggregatory concentration, PAC-1 - anti-integrin $\alpha \operatorname{IIb} \beta 3$ antibody, PE - phycoerythrin, PLC - phospholipase C, PRP - plateletrich plasma, $R_{\mathrm{M}}$ - chromatographic parameter, surrogate measurement of lipophilicity, TRAP-6 thrombin receptor-activating peptide (hexapeptide).

\section{REFERENCES}

1. P. Knekt, R. Jarvinen, A. Reunanen and J. Maatela, Flavonoid intake and coronary mortality in Finland: a cohort study, Brit. Med. J. 312 (1996) 478-481; https://doi.org/10.1136/bmj.312.7029.478

2. M. L. McCullough, J. J. Peterson, R. Patel, P. F. Jacques, R. Shah and J. T. Dwyer, Flavonoid intake and cardiovascular disease mortality in a prospective cohort of US adults, Am. J. Clin. Nutr. 95 (2012) 454-464; https://doi.org/10.3945/ajcn.111.016634 
3. M. L. Liang, X. W. Da, A. D. He, G. Q. Yao, W. Xie, G. Liu, J. Z. Xiang and Z. Y. Ming, Pentamethylquercetin (PMQ) reduces thrombus formation by inhibiting platelet function, Sci. Rep. 5 (2015) 11142 (11 pages); https://doi.org/10.1038/srep11142

4. Y. Wang, J. Tang, H. Zhu, X. Jiang, J. Liu, W. Xu, H. Ma, Q. Feng, J. Wu, M. Zhao and S. Peng, Aqueous extract of Rabdosia rubescens leaves: forming nanoparticles, targeting P-selectin, and inhibiting thrombosis, Int. J. Nanomed. 10 (2015) 6905-6918; https://doi.org/10.2147/IJN.S91316

5. S. Vaiyapuri, H. Roweth, M. S. Ali, A. J. Unsworth, A. R. Stainer, G. D. Flora, M. Crescente, C. I. Jones, L. A. Moraes and J. M. Gibbins, Pharmacological actions of nobiletin in the modulation of platelet function, Br. J. Pharmacol. 172 (2015) 4133-4145; https://doi.org/10.1111/bph.13191

6. E. S. Park, Y. Lim, S. H. Lee, B. M. Kwon, H. S. Yoo, J. T. Hong and Y. P. Yun, Antiplatelet activity of obovatol, a biphenolic component of Magnolia obovata, in rat arterial thrombosis and rabbit platelet aggregation, J. Atheroscler. Thromb. 18 (2011) 659-669; https://doi.org/10.5551/jat.7427

7. Y. R. Jin, X. H. Han, Y. H. Zhang, J. J. Lee, Y. Lim, J. H. Chung and Y. P. Yun, Antiplatelet activity of hesperetin, a bioflavonoid, is mainly mediated by inhibition of PLC-gamma2 phosphorylation and cyclooxygenase-1 activity, Atherosclerosis 194 (2007) 144-152; https://doi.org/10.1016/j.atherosclerosis.2006.10.011

8. P. Pignatelli, F. M. Pulcinelli, A. Celestini, L. Lenti, A. Ghiselli, P. P. Gazzaniga and F. Violi, The flavonoids quercetin and catechin synergistically inhibit platelet function by antagonizing the intracellular production of hydrogen peroxide, Am. J. Clin. Nutr. 72 (2000) 1150-1155; https://doi. org/10.1093/ajcn/72.5.1150

9. A. B. Hendrich, Flavonoid-membrane interactions: possible consequences for biological effects of some polyphenolic compounds, Acta. Pharmacol. Sin. 27 (2006) 27-40; https://doi.org/10.1111/j.1745-7254. 2006.00238.x

10. P. I. Oteiza, A. G. Erlejman, S. V. Verstraeten, C. L. Keen and C. G. Fraga, Flavonoid-membrane interactions: a protective role of flavonoids at the membrane surface? Clin. Dev. Immunol. 12 (2005) 19-25; https://doi.org/10.1080/10446670410001722168

11. T. Lhermusier, H. Chap and B. Payrastre, Platelet membrane phospholipid asymmetry: from the characterization of a scramblase activity to the identification of an essential protein mutated in Scott syndrome, J. Thromb. Haemost. 9 (2011) 1883-1891; https://doi.org/10.1111/j.1538-7836.2011.04478.x

12. C. A. Lipinski, F. Lombardo, B. W. Dominy and P. J. Feeney, Experimental and computational approaches to estimate solubility and permeability in drug discovery and development settings, Adv. Drug Deliv. Rev. 46 (2001) 3-26; https://doi.org/10.1016/S0169-409X(00)00129-0

13. G. L. Biagi, A. M. Barbaro, O. Gandolfi, M. C. Guerra and G. Cantelli-Forti, $R_{\mathrm{m}}$ values of steroids as an expression of their lipophilic character in structure-activity studies, J. Med. Chem. 18 (1975) 873883; https://doi.org/10.1021/jm00243a003

14. E. Kłosińska-Szmurło, F. A. Pluciński, M. Grudzień, K. Betlejewska-Kielak, J. Biernacka and A. P. Mazurek, Experimental and theoretical studies on the molecular properties of ciprofloxacin, norfloxacin, pefloxacin, sparfloxacin, and gatifloxacin in determining bioavailability, J. Biol. Phys. 40 (2014) 335-345; https://doi.org/10.1007/s10867-014-9354-z

15. B. J. Bennion, N. A. Be, M. W. McNerney, V. Lao, E. M. Carlson, C. A. Valdez, M. A. Malfatti, H. A. Enright, T. H. Nguyen, F. C. Lightstone and T. S. Carpenter, Predicting a drug's membrane permeability: A computational model validated with in vitro permeability assay data, J. Phys. Chem. B. 121 (2017) 5228-5237; https://doi.org/10.1021/acs.jpcb.7b02914

16. A. Daina, O. Michielin and V. Zoete, SwissADME: a free web tool to evaluate pharmacokinetics, drug-likeness and medicinal chemistry friendliness of small molecules, Sci. Rep. 7 (2017) 42717 (13 pages); https://doi.org/10.1038/srep42717

17. M. Bojić, Ž. Debeljak, M. Tomičić, M. Medić-Šarić and S. Tomić, Evaluation of antiaggregatory activity of flavonoid aglycone series, Nutrition J. 10 (2011) 73 (8 pages); https://doi.org/10.1186/1475-2891-10-73 
18. A. Brocchieri, L. Pacchiarini, A. Saporiti and G. Grignani, In vitro effect of verapamil on platelet activation induced by ADP, collagen or thrombin, Platelets 6 (1995) 195-199; https://doi. org/10.3109/09537109509078454

19. S. A. Saeed, H. Rasheed, F. A. Fecto, M. I. Achakzai, R. Ali, J. D. Connor and A. U. Gilani, Signalling mechanisms mediated by G-protein coupled receptors in human platelets, Acta Pharmacol. Sin. 25 (2004) 887-889.

20. J. W. Heemskerk, R. W. Farndale and S. O. Sage, Effects of U73122 and U73343 on human platelet calcium signalling and protein tyrosine phosphorylation, Biochim. Biophys. Acta 1355 (1997) 81-88; https://doi.org/10.1016/S0167-4889(96)00113-9

21. T. Wu, M. He, X. Zang, X. Zhou, T. Qiu, S. Pan and X. Xu, A structure-activity relationship study of flavonoids as inhibitors of E. coli by membrane interaction effect, Biochim. Biophys. Acta 1828 (2013) 2751-2756; https://doi.org/10.1016/j.bbamem.2013.07.029

22. M. Shichijo, N. Yamamoto, H. Tsujishita, M. Kimata, H. Nagai and T. Kokubo, Inhibition of syk activity and degranulation of human mast cells by flavonoids, Biol. Pharm. Bull. 26 (2003) 1685-1690; https://doi.org/10.1248/bpb.26.1685

23. A. Oliveira Filho, H. M. Fernandes, T. J. C. Assis, D. R. Meireles, O. Edeltrudes, E. Lima and H. L. F. Pêssoa, Pharmacological and toxicological analysis of flavonoid 5,7,4'-trimethoxyflavone: An in silico approach, IJPPR 7 (2015) 431-434.

24. C. van Dijk, A. J. Driessen and K. Recourt, The uncoupling efficiency and affinity of flavonoids for vesicles, Biochem. Pharmacol. 60 (2000) 1593-1600; https://doi.org/10.1016/S0006-2952(00)00488-3

25. A. Arora, T. M. Byrem, M. G. Nair and G. M. Strasburg, Modulation of liposomal membrane fluidity by flavonoids and isoflavonoids, Arch. Biochem. Biophys. 373 (2000) 102-109; https://doi.org/10.1006/ abbi.1999.1525

26. S. Kitagawa, M. Orinaka and H. Hirata, Depth-dependent change in membrane fluidity by phenolic compounds in bovine platelets and its relationship with their effects on aggregation and adenylate cyclase activity, Biochim. Biophys. Acta 1179 (1993) 277-282; https://doi.org/10.1016/0167-4889(93)90083-2

27. N. Vlasic, M. S. Medow, S. M. Schwarz, K. A. Pritchard and M. B. Stemerman, Lipid fluidity modulates platelet aggregation and agglutination in vitro, Life Sci. 53 (1993) 1053-1060; https://doi. org/10.1016/0024-3205(93)90258-5

28. K. Yoshida, S. Nagatoishi, D. Kuroda, N. Suzuki, T. Murata and K. Tsumoto, Phospholipid membrane fluidity alters ligand binding activity of a $\mathrm{G}$ protein-coupled receptor by shifting the conformational equilibrium, Biochemistry 58 (2019) 504-508; https://doi.org/10.1021/acs.biochem.8b01194 (in press)

29. M. Bojić, Ž. Debeljak, M. Medić-Šarić and M. Tomičić, Interference of selected flavonoid aglycons in platelet aggregation assay, Clin. Chem. Lab. Med. 50 (2012) 1403-1408; https://doi.org/10.1515/cclm2011-0960

30. L. M. Ostertag, N. O'Kennedy, G. W. Horgan, P. A. Kroon, G. G. Duthie and B. de Roos, In vitro anti-platelet effects of simple plant-derived phenolic compounds are only found at high, non-physiological concentrations, Mol. Nutr. Food Res. 55 (2011) 1624-1636; https://doi.org/10.1002/ mnfr.201100135

31. S. Vaiyapuri, M. S. Ali, L. A. Moraes, T. Sage, K. R. Lewis, C. I. Jones and J. M. Gibbins, Tangeretin regulates platelet function through inhibition of phosphoinositide 3-kinase and cyclic nucleotide signalling, Arterioscler. Thromb. Vasc. Biol. 33 (2013) 2740-2749; https://doi.org/10.1161/ATVBAHA.113.301988

32. T. Gremmel, R. Koppensteiner and S. Panzer, Comparison of aggregometry with flow cytometry for the assessment of agonists'-induced platelet reactivity in patients on dual antiplatelet therapy, PLoS One 10 (2015) e0129666 (13 pages); https://doi.org/10.1371/journal.pone.0129666

33. K. Koltai, G. Kesmarky, G. Feher, A. Tibold and K. Toth, Platelet aggregometry testing: Molecular mechanisms, techniques and clinical implications, Int. J. Mol. Sci. 18 (2017) 1803 (21 pages); https:// doi.org/10.3390/ijms18081803 